\title{
A symplectic subgroup of a pseudounitary group as a subset of Clifford algebra
}

\author{
Nikolai Marchuk, Roman Dyabirov
}

March 31, 2022

\begin{abstract}
Let $\mathrm{Cl} 1(1,3)$ and $\mathrm{Cl} 2(1,3)$ be the subsets of elements of the Clifford algebra $\mathrm{Cl}(1,3)$ of ranks 1 and 2 respectively. Recently it was proved that the subset $\mathrm{Cl} 2(\mathrm{p}, \mathrm{q})+\mathrm{iCl1}(\mathrm{p}, \mathrm{q})$ of the complex Clifford algebra can be considered as a Lie algebra. In this paper we prove that for $p=1, q=3$ the Lie algebra $\mathrm{Cl} 2(\mathrm{p}, \mathrm{q})+\mathrm{iCl}(\mathrm{p}, \mathrm{q})$ is isomorphic to the well known matrix Lie algebra $\mathrm{sp}(4, \mathrm{R})$ of the symplectic Lie group $\operatorname{Sp}(4, \mathrm{R})$. Also we define the so called symplectic group of Clifford algebra and prove that this Lie group is isomprphic to the symplectic matrix group $\mathrm{Sp}(4, \mathrm{R})$.
\end{abstract}

\section{Introduction}

In [1] was considered the complex Clifford algebras $\mathcal{C}(p, q)$ and was found subsets of Clifford algebra closed with respect to the commutator $[U, V]=U V-V U$. For all positive integer $p, q(p+q \geq 2)$ the subset $\mathcal{C} \ell_{2}^{\mathbb{R}}(p, q) \oplus i \mathcal{C} \ell_{1}^{\mathbb{R}}(p, q)$ of Clifford algebra $\mathcal{C}(p, q)$ can be considered as a Lie algebra. In this paper we prove that for $p=1, q=3$ the Lie algebra $\mathcal{C} \ell_{2}^{\mathbb{R}}(p, q) \oplus i \mathcal{C} \ell_{1}^{\mathbb{R}}(p, q)$ is isomorphic to the well known matrix Lie algebra $\operatorname{sp}(4, R)$ of the symplectic Lie group $\operatorname{Sp}(4, R)$. Also we define the so called symplectic group of Clifford algebra and prove that this Lie group is isomprphic to the symplectic matrix group $\operatorname{Sp}(4, R)$. 


\section{Clifford algebras $\mathcal{C}(p, q)$}

Let $\mathrm{F}$ be the field of real numbers $\mathbb{R}$ or the field of complex numbers $\mathbb{C}$ and let $n$ be a natural number. Consider $2^{n}$ dimensional vector space $\mathcal{E}$ over the field $\mathbb{F}$ with a basis

$$
e, e^{a}, e^{a_{1} a_{2}}, \ldots, e^{1 \ldots n}, \quad a_{1}<a_{2}<\cdots,
$$

with elements numbered dy ordered multi-indices of length from 0 to $n$. Indices $a, a_{1}, \ldots$ take values from 1 to $n$.

Let $p, q$ be nonnegative integer numbers and $p+q=n$. Consider a diagonal matrix of dimension $n$

$$
\eta=\eta(p, q)=\operatorname{diag}(1, \ldots, 1,-1, \ldots,-1),
$$

with $p$ pieces of 1 and $q$ pieces of -1 on the diagonal. By $\eta^{a b}=\eta_{a b}$ denote elements of $\eta$.

Following rules define product $U, V \rightarrow U V$ of elements of the vector space $\mathcal{E}$ :

1. For any $U, V, W \in \mathcal{E}$

$$
\begin{aligned}
& U(V+W)=U V+U W, \quad(U+V) W=U W+V W \\
& (U V) W=U(V W) ;
\end{aligned}
$$

2. $e U=U e=U$, for any $U \in \mathcal{E}$;

3. $e^{a} e^{b}+e^{b} e^{a}=2 \eta^{a b} e$, for $a, b=1, \ldots, n$;

4. $e^{a_{1}} \ldots e^{a_{k}}=e^{a_{1} \ldots a_{k}}$, for $1 \leq a_{1}<\cdots<a_{k} \leq n$.

The operation of multiplication converts vector space $\mathcal{E}$ to an algebra. This algebra is called the Clifford algebra and denoted by $\mathcal{C} \ell^{\mathbb{F}}(p, q)$ (if $\mathbb{F}=\mathbb{C}$, then $\left.\mathcal{C}(p, q)=\mathcal{C} \ell^{\mathbb{C}}(p, q)\right)$. For $p=n, q=0$ we use notation $\mathcal{C} \ell^{\mathbb{F}}(n)=\mathcal{C} \ell^{\mathbb{F}}(n, 0)$. Elements $e^{a}$ are called generators of Clifford algebra $\mathcal{C} \ell^{\mathbb{F}}(p, q) \cdot \mathcal{C} \ell^{\mathbb{R}}(p, q)$ is real Clifford algebra and $\mathcal{C}(p, q)$ is complex Clifford algebra. 
Any element $U \in \mathcal{C} \ell^{\mathbb{F}}(p, q)$ can be written in the form

$$
U=u e+u_{a} e^{a}+\sum_{a_{1}<a_{2}} u_{a_{1} a_{2}} e^{a_{1} a_{2}}+\ldots+u_{1 \ldots n} e^{1 \ldots n}
$$

with coefficients $u, u_{a}, u_{a_{1} a_{2}}, \ldots, u_{1 \ldots n} \in \mathbb{F}$, which numbered by ordered multi-indices of length from 0 to $n$.

Denote by $\mathcal{C} \ell_{k}^{\mathbb{F}}(p, q), k=0, \ldots n$, subspaces of the vector space $\mathcal{C} \ell^{\mathbb{F}}(p, q)$ that span over basis elements $e^{a_{1} \ldots a_{k}}$. Elements of $\mathcal{C} \ell_{k}^{\mathbb{F}}(p, q)$ are called elements of rank $k$. Sometimes it is suitable to denote $\stackrel{k}{U} \in \mathcal{C} \ell_{k}^{\mathbb{F}}(p, q)$. We have

$$
\mathcal{C} \ell^{\mathbb{F}}(p, q)=\mathcal{C} \ell_{0}^{\mathbb{F}}(p, q) \oplus \ldots \oplus \mathcal{C} \ell_{n}^{\mathbb{F}}(p, q)=\mathcal{C} \ell_{\text {even }}^{\mathbb{F}}(p, q) \oplus \mathcal{C} \ell_{\text {odd }}^{\mathbb{F}}(p, q),
$$

where

$$
\begin{aligned}
\mathcal{C} \ell_{\text {even }}^{\mathbb{F}}(p, q) & =\mathcal{C} \ell_{0}^{\mathbb{F}}(p, q) \oplus \mathcal{C} \ell_{2}^{\mathbb{F}}(p, q) \oplus \ldots \\
\mathcal{C} \ell_{\text {odd }}^{\mathbb{F}}(p, q) & =\mathcal{C} \ell_{1}^{\mathbb{F}}(p, q) \oplus \mathcal{C} \ell_{3}^{\mathbb{F}}(p, q) \oplus \ldots
\end{aligned}
$$

and

$$
\operatorname{dim} \mathcal{C} \ell_{k}^{\mathbb{F}}(p, q)=C_{n}^{k}, \quad \operatorname{dim} \mathcal{C} \ell_{\text {even }}^{\mathbb{F}}(p, q)=\operatorname{dim} \mathcal{C} \ell_{\text {odd }}^{\mathbb{F}}(p, q)=2^{n-1},
$$

$C_{n}^{k}$ are binomial coefficients. Let us take the antisymmetric coefficients $u_{a_{1} \ldots a_{k}}=$ $u_{\left[a_{1} \ldots a_{k}\right]}$. Consider an element

$$
\stackrel{k}{U}=\sum_{a_{1}<\cdots<a_{k}} u_{a_{1} \ldots a_{k}} e^{a_{1} \ldots a_{k}}=\sum_{a_{1}<\cdots<a_{k}} u_{a_{1} \ldots a_{k}} e^{a_{1}} \ldots e^{a_{k}}
$$

We have

$$
\stackrel{k}{U}=\frac{1}{k !} u_{a_{1} \ldots a_{k}} e^{a_{1}} \ldots e^{a_{k}}=\frac{1}{k !} u_{a_{1} \ldots a_{k}} e^{a_{1}} \wedge \ldots \wedge e^{a_{k}}
$$

for $\mathrm{U}$ from (3). 


\section{A Hermitian conjugation operation}

Using the projection operation onto the one-dimensional subspace $\mathcal{C l}_{0}(p, q)$, we get a trace of an element $U \in \mathcal{C}(p, q)$

$$
\operatorname{Tr}: \mathcal{C} \ell(p, q) \rightarrow \mathbb{C}
$$

where

$$
\operatorname{Tr}\left(u e+u_{a} e^{a}+\ldots\right)=u .
$$

This operation has the following properties:

$$
\operatorname{Tr}(U V)=\operatorname{Tr}(V U), \quad \operatorname{Tr}([U, V])=0 .
$$

Now we introduce the structure of unitary (Euclidian) space on Clifford algebra [1. Let $\dagger: \mathcal{C} \ell^{\mathbb{F}}(p, q) \rightarrow \mathcal{C} \ell^{\mathbb{F}}(p, q)$ be a linear Hermitian conjugation operation of the Clifford algebra elements. Following rules define a Hermitian conjugation operation of Clifford algebra elements:

$$
\begin{gathered}
\left(e^{i_{1} \ldots i_{k}}\right)^{\dagger}=e_{i_{k}} \ldots e_{i_{1}}, \quad \text { and } \quad \lambda^{\dagger}=\bar{\lambda}, \quad \forall \lambda \in \mathbb{C}, \\
(U+V)^{\dagger}=V^{\dagger}+U^{\dagger} \quad \forall U, V \in \mathcal{C} \ell^{\mathbb{F}}(p, q),
\end{gathered}
$$

where $e_{a}=\eta_{a b} e^{b}$. We say that $\dagger: \mathcal{C} \ell^{\mathbb{F}}(p, q) \rightarrow \mathcal{C} \ell^{\mathbb{F}}(p, q)$ is the operation of Hermitian conjugation of Clifford algebra elements. It is easy to see that

$$
(U V)^{\dagger}=V^{\dagger} U^{\dagger}, \quad U^{\dagger \dagger}=U
$$

Now we can define a Hermitian (Euclidian) scalar product of Clifford algebra elements by the formula

$$
(U, V)=\operatorname{Tr}\left(V^{\dagger} U\right)
$$

In this case we have

$$
\left(e^{i_{1} \ldots i_{k}}, e^{i_{1} \ldots i_{k}}\right)=\operatorname{Tr}\left(e_{i_{k}} \ldots e_{i_{1}} e^{i_{1}} \ldots e^{i_{k}}\right)=\operatorname{Tr}(e)=1,
$$


(no summation w.r.t. $\left.i_{1}, \ldots, i_{k}\right)$. Basis (11) of $\mathcal{C} \mathbb{F}^{\mathbb{F}}(p, q)$ is orthonormal with respect to this scalar product and property $(7)$ is valid

$$
(U, U)=\sum_{k=0}^{n} \sum_{a_{1}<\ldots<a_{k}}\left|u_{a_{1} \ldots a_{k}}\right|^{2}>0 .
$$

For generators $e^{a}$ formula (6) gives

$$
\begin{aligned}
& \left(e^{a}\right)^{\dagger}=e^{a} \text { for } a=1, \ldots, p ; \\
& \left(e^{a}\right)^{\dagger}=-e^{a} \text { for } a=p+1, \ldots, n .
\end{aligned}
$$

\section{$3 \quad$ Pseudounitary groups of Clifford algebras}

Let $r, s$ be nonnegative integers, $r+s>0$. Consider a Lie group of pseudounitary matrices and a Lie group of special pseudounitary matrices:

$$
\begin{aligned}
\mathrm{U}(r, s) & =\left\{U \in \operatorname{Mat}(n, \mathbb{C}): U^{\dagger} \beta U=\beta\right\}, \\
\mathrm{SU}(r, s) & =\{U \in \mathrm{U}(r, s): \operatorname{det} U=1\},
\end{aligned}
$$

where $n=r+s$ and $\beta=\operatorname{diag}(1, \ldots, 1,-1, \ldots,-1)$ is a diagonal matrix with $r$ pieces of 1 and $s$ pieces of -1 on the diagonal.

Now let us consider the four sets of Clifford algebra elements:

$$
\begin{aligned}
\mathrm{W}(\mathcal{C}(p, q)) & =\left\{U \in \mathcal{C} \ell(p, q): U^{*} U=e\right\}, \\
\mathrm{SW}(\mathcal{C}(p, q)) & =\{U \in \mathrm{W}(\mathcal{C}(p, q)): \operatorname{Det} U=1\}, \\
\mathrm{w}(\mathcal{C l}(p, q)) & =\left\{U \in \mathcal{C}(p, q): U^{*}=-U\right\}, \\
\mathrm{Sw}(\mathcal{C l}(p, q)) & =\{U \in \mathrm{w}(\mathcal{C}(p, q)): \operatorname{Tr} U=0\} .
\end{aligned}
$$

Note that the sets $\mathrm{W}(\mathcal{C} \ell(p, q))$ and $\mathrm{SW}(\mathcal{C}(p, q))$ are closed with respect to the Clifford product and the sets $\mathrm{w}(\mathcal{C}(p, q))$ and $\operatorname{sw}(\mathcal{C}(p, q))$ are closed with respect to the commutator. The sets $\mathrm{W}(\mathcal{C}(p, q))$ and $\mathrm{SW}(\mathcal{C}(p, q))$ can be considered as Lie groups 
and the sets $\mathrm{w}(\mathcal{C}(p, q))$ and $\mathrm{sw}(\mathcal{C}(p, q))$ can be considered as Lie algebras of the Lie groups $\mathrm{W}(\mathcal{C l}(p, q))$ and $\mathrm{SW}(\mathcal{C l}(p, q))$.

The group $\mathrm{W}(\mathcal{C}(p, q))$ is called the pseudounitary group of Clifford algebra $\mathcal{C}(p, q)$. The group $\mathrm{SW}(\mathcal{C}(p, q))$ is called the special pseudounitary group of Clifford algebra.

\section{Symplectic Lie groups and their Lie algebras}

Let us consider a real symplectic Lie group of $n \times n$-matrices, $n=2 m$ and let us consider the real Lie algebra of this group

$$
\begin{aligned}
& \operatorname{Sp}(n, \mathbb{R})=\left\{U \in \operatorname{Mat}(n, \mathbb{R}): U^{T} J U=J\right\}, \\
& \operatorname{sp}(n, \mathbb{R})=\left\{u \in \operatorname{Mat}(n, \mathbb{R}): u^{T} J=-J u\right\},
\end{aligned}
$$

where $U^{T}$ is a transpose matrix, $J$ is the matrix

$$
J=\left(\begin{array}{cc}
0 & -I_{m} \\
I_{m} & 0
\end{array}\right)
$$

and $I_{m}$ is an identity $m \times m$ - matrix. Note that $J^{2}=-\mathbf{1}(\mathbf{1}$ is the identity $4 \times 4$ matrix). Mostafazadeh 4 shows that the symplectic group $\operatorname{sp}(2 n, R)$ is a subgroup of the pseudounitary group $\mathrm{U}(n, n)$.

\section{A symplectic group of Clifford algebra}

Now define the two sets of Clifford algebra elements

$$
\begin{aligned}
& \operatorname{Sp}(\mathcal{C l}(1,3))=\left\{V \in \mathcal{C} l_{\text {even }}^{\mathbb{R}}(1,3) \oplus i \mathcal{C} \ell_{\text {odd }}^{\mathbb{R}}(1,3): V^{*} V=e\right\}, \\
& \operatorname{sp}(\mathcal{C l}(1,3))=\left\{v \in i \mathcal{C l}_{1}^{\mathbb{R}}(1,3) \oplus \mathcal{C l}_{2}^{\mathbb{R}}(1,3)\right\} .
\end{aligned}
$$

We have $\operatorname{Sp}(\mathcal{C}(1,3)) \subset \mathrm{W}(\mathcal{C}(1,3))$. Suppose that this set is closed with respect to the product; then $\operatorname{Sp}(\mathcal{C l}(1,3))$ can be considered as a group (the Lie group) 
with respect to the product. This group is called the symplectic group of Clifford algebra $\mathrm{Cl}(1,3)$. The relation of this group with the matrix symplectic group will be explained in Theorem 1 .

In [1] we see that the set $\operatorname{sp}(\mathcal{C}(1,3)) \subset \mathrm{w}(\mathcal{C}(1,3))$ is closed with respect to the commutator $[u, v]=u v-v u$. So $\operatorname{sp}(\mathcal{C l}(1,3))$ is a Lie algebra with respect to the commutator.

Theorem 1 . The group $\operatorname{Sp}(\mathcal{C}(1,3))$ is isomorphic to the group $\operatorname{Sp}(4, \mathbb{R})$ and the Lie algebra $\mathrm{sp}(\mathcal{C}(1,3))$ is isomorphic to the Lie algebra $\mathrm{sp}(4, \mathbb{R})$ :

$$
\begin{aligned}
& \operatorname{Sp}(\mathcal{C}(1,3)) \simeq \operatorname{Sp}(4, \mathbb{R}) \\
& \operatorname{sp}(\mathcal{C}(1,3)) \simeq \operatorname{sp}(4, \mathbb{R}) .
\end{aligned}
$$

Proof. It is well known $[3$ that the complex Clifford algebra $\mathcal{C}(1,3)$ is isomorphic to the algebra of complex matrices $\operatorname{Mat}(4, \mathbb{C})$. Let $e^{a}, a=0,1,2,3$ be generators and $e$ be the identity element of Clifford algebra $\mathrm{C}(1,3)$. Consider the matrix representation $\gamma$ of Clifford algebra elements

$$
\gamma: \mathcal{C}(1,3) \rightarrow \operatorname{Mat}(4, \mathbb{C}),
$$

such that

$$
\begin{aligned}
\gamma(A+B) & =\gamma(A)+\gamma(B) \\
\gamma(\lambda A) & =\lambda \gamma(A), \\
\gamma(A B) & =\gamma(A) \gamma(B), \\
\gamma(e) & =\mathbf{1},
\end{aligned}
$$

where $A, B$ are arbitrary elements of $\mathcal{C}(1,3) ; \lambda$ is a complex number; $\mathbf{1}$ is the identity 
$4 \times 4$-matrix and generators of Clifford algebra represented by the following matrices:

$$
\begin{aligned}
\gamma\left(e^{0}\right) & =\left(\begin{array}{cccc}
0 & 0 & i & 0 \\
0 & 0 & 0 & i \\
-i & 0 & 0 & 0 \\
0 & -i & 0 & 0
\end{array}\right), & \gamma\left(e^{1}\right) & =\left(\begin{array}{cccc}
0 & -i & 0 & 0 \\
-i & 0 & 0 & 0 \\
0 & 0 & 0 & i \\
0 & 0 & i & 0
\end{array}\right), \\
\gamma\left(e^{2}\right) & =\left(\begin{array}{llll}
0 & 0 & i & 0 \\
0 & 0 & 0 & i \\
i & 0 & 0 & 0 \\
0 & i & 0 & 0
\end{array}\right), & \gamma\left(e^{3}\right) & =\left(\begin{array}{cccc}
-i & 0 & 0 & 0 \\
0 & i & 0 & 0 \\
0 & 0 & i & 0 \\
0 & 0 & 0 & -i
\end{array}\right) .
\end{aligned}
$$

We say that the matrix representation $\gamma$ is a modified Majorana's representation. Let us remind that the representation $\gamma$ defines the isomorphism of $\mathcal{C}(1,3)$ and $\operatorname{Mat}(4, \mathbb{C})$.

Generators $e^{a}$ of Clifford algebra satisfy the following formulas

$$
e^{a} e^{b}+e^{b} e^{a}=2 \eta^{a b} e, \quad a, b=0,1,2,3 .
$$

So the matrices $\gamma^{a}=\gamma\left(e^{a}\right), a=0,1,2,3$ satisfy the conditions

$$
\gamma^{a} \gamma^{b}+\gamma^{b} \gamma^{a}=2 \eta^{a b} \mathbf{1}, \quad a, b=0,1,2,3 .
$$

Note that the sixteen matrices

$$
\mathbf{1}, \gamma^{a}, \gamma^{a} \gamma^{b}, \gamma^{a} \gamma^{b} \gamma^{c}, \gamma^{0} \gamma^{1} \gamma^{2} \gamma^{3}, \quad a<b<\ldots
$$

are linearly independent and form the basis of the matrix algebra Mat $(4, \mathbb{C})$.

Note also that the matrices $\gamma^{a}$ satisfy to the conditions

$$
\left(\gamma^{0}\right)^{\dagger}=\gamma^{0}, \quad\left(\gamma^{k}\right)^{\dagger}=-\gamma^{k}, \quad k=1,2,3 .
$$

Therefore the representation $\gamma$ is consistent with the Hermitian conjugation operation

$$
\gamma(A)^{\dagger}=\gamma\left(A^{\dagger}\right),
$$

where $\gamma(A)^{\dagger}$ is the Hermition conjugated matrix, and $A^{\dagger}=e^{0} A^{*} e^{0}$ is the Hermitian conjugated element of Clifford algebra $\mathrm{Cl}(1,3)$. 
Denote by $\gamma^{-1}$ the inverse map

$$
\gamma^{-1}: \operatorname{Mat}(4, \mathbb{C}) \rightarrow \mathcal{C}(1,3) .
$$

Matrices $i \gamma^{a}$ are real, therefore the maps $\gamma, \gamma^{-1}$ give the isomorphism

$$
\mathcal{C} \ell_{\text {even }}^{\mathbb{R}}(1,3) \oplus i \mathcal{C} \ell_{\text {odd }}^{\mathbb{R}}(1,3) \simeq \operatorname{Mat}(4, \mathbb{R}) .
$$

Our the representation $\gamma$ is such that

$$
J=i \gamma\left(e^{0}\right)
$$

where the matrix $J$ is used in the definition of the group of symplectic matrices.

For any $A \in \mathcal{C}(1,3)$ we have

$$
A^{\dagger}=e^{0} A^{*} e^{0}, \text { or } A^{*}=e^{0} A^{\dagger} e^{0} .
$$

Therefore for any element $A \in \mathcal{C} \ell_{\text {even }}^{\mathbb{R}}(1,3) \oplus i \mathcal{C} \ell_{\text {odd }}^{\mathbb{R}}(1,3)$ we have

$$
\gamma\left(A^{*}\right)=\gamma\left(e^{0}\right) \gamma\left(A^{\dagger}\right) \gamma\left(e^{0}\right)=-J \gamma(A)^{T} J
$$

Consider the definition of group $\operatorname{Sp}(\mathcal{C}(1,3))$. The condition $A^{*} A=e$ leads to the relation

$$
\gamma\left(A^{*}\right) \gamma(A)=\gamma(e) .
$$

Using this relation and conditions

$$
\gamma\left(A^{*}\right)=-J \gamma(A)^{T} J, \quad J^{2}=-\mathbf{1}
$$

we get

$$
\gamma(A)^{T} J \gamma(A)=J
$$

From the definition of the group $\operatorname{Sp}(4, \mathbb{R})$ it follows that the group $\operatorname{Sp}(\mathcal{C}(1,3))$ is isomorphic to the group $\operatorname{Sp}(4, \mathbb{R})$

$$
\operatorname{Sp}(\mathcal{C}(1,3)) \simeq \operatorname{Sp}(4, \mathbb{R})
$$


Let us remind the definition of Lie algebra $\mathrm{w}(\mathcal{C}(1,3))$ of the pseudounitary group $\mathrm{W}(\mathcal{C}(1,3))$

$$
\mathrm{w}(\mathcal{C}(1,3))=\left\{w \in \mathcal{C} \ell(1,3): w^{*}=-w\right\} .
$$

This definition is equivalent to the following:

$$
\mathrm{w}(\mathcal{C l}(1,3))=\left\{w \in i \mathcal{C} \ell_{0}^{\mathbb{R}}(1,3) \oplus i \mathcal{C} l_{1}^{\mathbb{R}}(1,3) \oplus \mathcal{C} l_{2}^{\mathbb{R}}(1,3) \oplus \mathcal{C} l_{3}^{\mathbb{R}}(1,3) \oplus i \mathcal{C}_{4}^{\mathbb{R}}(1,3)\right\} .
$$

Note that the definition of Lie algebra $\operatorname{sp}(\mathcal{C}(1,3))$ can be written in the form

$$
\operatorname{sp}(\mathcal{C}(1,3))=\left\{w \in \mathrm{w}(\mathcal{C}(1,3)): w \in \mathcal{C}_{\text {even }}^{\mathbb{R}}(1,3) \oplus i \mathcal{C}_{\text {odd }}^{\mathbb{R}}(1,3)\right\} .
$$

Hence, $\operatorname{sp}(\mathcal{C}(1,3))$ is the intersection of following sets:

$$
\begin{aligned}
& \left\{i \mathcal{C} \ell_{0}^{\mathbb{R}}(1,3) \oplus i \mathcal{C} \ell_{1}^{\mathbb{R}}(1,3) \oplus \mathcal{C} \ell_{2}^{\mathbb{R}}(1,3) \oplus \mathcal{C} \ell_{3}^{\mathbb{R}}(1,3) \oplus i \mathcal{C} \ell_{4}^{\mathbb{R}}(1,3)\right\} \\
& \cap\left\{\mathcal{C} \mathcal{C}_{\text {even }}^{\mathbb{R}}(1,3) \oplus i \mathcal{C} \ell_{\text {odd }}^{\mathbb{R}}(1,3)\right\} .
\end{aligned}
$$

Any element of the Lie algebra $w \in \mathcal{C} \mathcal{C}_{\text {even }}^{\mathbb{R}}(1,3) \oplus i \mathcal{C}_{\text {odd }}^{\mathbb{R}}(1,3)$ represents as a real matrix. Therefore using the matrix representation $\gamma$ for the expression $w^{*}=-w$ we get the relations

$$
-J \gamma(w)^{T} J=-\gamma(w), \quad \text { or } \quad \gamma(w)^{T} J=-J \gamma(w) .
$$

It is clear that the last expression defines the Lie algebra $\operatorname{sp}(4, \mathbb{R})$. Hence the Lie algebra $\operatorname{sp}(\mathcal{C}(1,3))$ is isomorphic to the Lie algebra $\operatorname{sp}(4, \mathbb{R})$. This completes the proof.

\section{References}

[1] Marchuk N. G., Shirokov D. S. Unitary spaces on Clifford algebras, Adv. appl. Clifford alg., 18, (2008), pp.237-254, arXiv:0705.1641v1 [math-ph], (2007).

[2] Hestenes D., Space-Time Algebra, Gordon and Breach, New York, (1966).

[3] Lounesto P., Clifford Algebras and Spinors, Cambridge Univ. Press (1997, 2001)

[4] Mostafazadeh A., Pseudo-Unitary Operators and Pseudo-Unitary Quantum Dynamics, arXiv:0302050v2 [math-ph], (2003). 